\title{
On Secure Digital Image Watermarking Techniques
}

\author{
Manjit Thapa $^{1}$, Sandeep Kumar Sood ${ }^{2 *}$ \\ ${ }^{1}$ Department of Computer Science, Sri Sai College of Engineering \& Technonogy, Badhani, Pathankot, India \\ ${ }^{2}$ Department of Computer Science and Engineering, G.N.D.U.R.C., Gurdaspur, Punjab, India \\ E-mail:manjit.thapa@yahoo.co.in, san1198@gmail.com \\ Received July 29, 2011; revised September 9, 2011; accepted September 20, 2011
}

\begin{abstract}
Digital watermarking is used to hide the information inside a signal, which can not be easily extracted by the third party. Its widely used application is copyright protection of digital information. It is different from the encryption in the sense that it allows the user to access, view and interpret the signal but protect the ownership of the content. One of the current research areas is to protect digital watermark inside the information so that ownership of the information cannot be claimed by third party. With a lot of information available on various search engines, to protect the ownership of information is a crucial area of research. In latest years, several digital watermarking techniques are presented based on discrete cosine transform (DCT), discrete wavelets transform (DWT) and discrete fourier transforms (DFT). In this paper, we propose an algorithm for digital image watermarking technique based on singular value decomposition; both of the $\mathrm{L}$ and $\mathrm{U}$ components are explored for watermarking algorithm. This technique refers to the watermark embedding algorithm and watermark extracting algorithm. The experimental results prove that the quality of the watermarked image is excellent and there is strong resistant against many geometrical attacks.
\end{abstract}

Keywords: Digital Image Watermarking, Singular Value Decomposition, Watermark Embedding Algorithm, Watermark Extracting Algorithm, Ratio Analysis, Security Analysis

\section{Introduction}

Digital watermarking is a technique that embeds data called watermark into a multimedia object so that watermark can be detected to make an assertion about the objects. It can be categorized as visible or invisible. Example of visible watermarking is the logo visible superimposed on the corner of television channel in a television picture. On the other hand, invisible watermark is hidden in the object, which can be detected by an authorized person. Such watermarks are used for suit the author authentication and detecting unauthorized copying. The novel technology of digital watermarking has been sponsored by many consultants as the best method for such multimedia copyright protection problem $[1,2]$. Digital watermarking is having a variety of useful applications such as digital cameras, medical imaging, image databases, video on demand systems, and many others. In recent years, many digital image watermarking techniques have been proposed in the literature which is based on spatial domain technique and frequency domain technique. These techniques are used in watermark em- bedding algorithm and watermark extracting algorithm. In 2002, Ali [3] proposed an approach based on DWT and DCT to improve the performance of the DWT-based watermarking algorithms. In this method, watermarking is done by embedding the watermark in first and second level of DWT sub-bands of the host image, followed by the application of DCT on the selected DWT sub-bands. The combination of these two transforms improved the watermarking performance considerably in comparison with only watermarking approaches. They showed that the quality of watermark image is very good. In 2005, Chen [4] proposed a singular value decomposition scheme based on components of $\mathrm{D}$ and $\mathrm{U}$ without using DWT, DCT and DFT transforms. They showed that quality of watermarked image is good on their schemes. In 2007, Seed [5] introduced a novel digital watermarking method based on single key image for extracting different watermarks. In this method, they used Arnold transform technique in watermark embedding and extraction, which is based on DWT and DCT algorithm. With the popularity of internet and availability of large storage devices, storing and transferring an image is 
simple and feasible. They showed that robustness of the algorithm against many signal processing operations. In 2010, Lamma and Ali [6] suggested two blind, imperceptible and robust video watermarking algorithms that are based on singular value decomposition. Each algorithm integrates the watermark in the transform domain. They used the components of matrices such as $\mathrm{U}$ and $\mathrm{V}$. Their schemes are shown to provide very good performance in watermarked video as compared to Chan [4].

Most of the domain transformation watermarking techniques works with DCT and DWT. However singular value decomposition (SVD) is one of the most powerful numeric analysis techniques and used in various requirements. These requirements can be organized and described as follows [7-10].

Undeletable: An embedded watermark is difficult to detect and cannot be removed by an illegal person. Also the algorithm must resist different attacks.

Perceptually visible: The original images and watermarked images cannot be distinguished by the human eye. This means that there is not enough alteration of a watermarked image to prevent motivation to an illegal person.

Unambiguous: An embedded watermark selected from a watermarked image that must be clear enough for ownership to be determined. In this way, the extracted watermark cannot be distorted to such an extent that the original watermark cannot be recognized.

In this paper, we will describe a digital image watermarking algorithm based on singular value decomposition technique. This paper is organized as follows. In Section 2, we introduce the SVD transformation and SVD based watermarking techniques briefly. In Section 3 , we propose the embedding and extracting algorithm. In Section 4, we evaluate the performance of watermark image. In section 5, we show the experimental results and Section 6 conclude the paper.

\section{A Review of Related Work}

Singular value decomposition (SVD) is a mathematical technique based on linear algebra and used by factorization of a real matrix or complex matrix, with many useful applications in signal processing and statistics.

\subsection{Singular Value Decomposition (SVD)}

Singular value decomposition is one of a number of valuable numerical analysis tools which is used to analyze matrices. It can be appeared from three jointly compatible points of view. On the other hand, we can see it as a method for transforming correlated variables into a set of uncorrelated ones that better expose the various relationships among the original data items. At the same time, SVD is a method for identifying and ordering the dimensions along which data points demonstrate the most variation. This attach the third way of viewing singular value decomposition, which accepted the most variation, it's possible to find the best approximation of the original data points using less dimensions. Hence, SVD can be seen as a method for data reduction. In SVD transformation, a matrix can be decayed into three matrices that are having the same size as the original matrix. It is useful to establish a contrast with Gaussian elimination and its equation. Given $\mathrm{A}$ is a $n \times n$ square matrix, this matrix can be decomposed into three components, $\mathrm{L}$, $\mathrm{D}$ and $\mathrm{U}$, respectively such that

$$
\begin{gathered}
\text { [L D U }=\operatorname{SVD}(\mathrm{A}), \mathrm{A}^{\prime}=\mathrm{LDU}^{\mathrm{T}}, \mathrm{L}^{-1} \text { where A }=\mathrm{LDU} \text {. } \\
\left(\begin{array}{lll}
l_{1,1} & l_{1,2} & l_{1, n} \\
l_{2,1} & l_{2,2} & l_{2, n} \\
l_{3,1} & l_{3,2} & l_{3, n}
\end{array}\right)\left(\begin{array}{lll}
\sigma_{1,1} & \sigma_{1,2} & \sigma_{1, n} \\
\sigma_{2,1} & \sigma_{2,2} & \sigma_{2, n} \\
\sigma_{3,1} & \sigma_{3,2} & \sigma_{3, n}
\end{array}\right) \\
\left(\begin{array}{lll}
u_{1,1} & u_{1,2} & u_{1, n} \\
u_{2,1} & u_{2,2} & u_{2, n} \\
u_{3,1} & u_{3,2} & u_{3, n}
\end{array}\right)=\sum_{i=1}^{n} \sigma_{i} l_{i} u_{i}^{\mathrm{T}}
\end{gathered}
$$

where the $\mathrm{L}$ and $\mathrm{U}$ components are real unitary matrices or complex matrices with small singular values, and the D component is an $n \times n$ diagonal matrix with larger singular value or eigen vector values entries which specify $\sigma_{1,1}>\sigma_{2,2}>\cdots \sigma_{k, k, k+1}=\ldots \sigma_{n, n}=\mathbf{0}$. $\sum$ are non zero matrix by diagonals of A. SVD is nonlinear because the orthogonal matrices $\mathrm{L}$ and $\mathrm{U}$ depend on $\mathrm{A}$ and shown in Equation (1). $\mathrm{A}^{\prime}$ is the reconstructed matrix after the inverse SVD transformation. Reduced singular value decomposition is the mathematical technique underlying a type of document retrieval and word semblance method. These are also known as Latent Semantic Indexing or Latent Semantic Analysis. In this way, the three components of matrices L, D, and U specify $\mathbf{A} \boldsymbol{u}_{i}=\sigma_{i} \mathbf{l}_{i}$ and $\boldsymbol{\mu}_{i}^{\mathrm{T}} \mathbf{A}$ $=\sigma_{i} u_{i}{ }^{\mathrm{T}}$.

\section{SVD Example}

A matrix is said to be square if it has the same number of rows as columns. To designate the size of a square matrix with $\mathrm{n}$ rows and columns, it is called n-square matrix. For example, the matrix below is 3-square. As an example to simplify SVD transformation, suppose

$$
A=\left(\begin{array}{ccc}
10 & 21 & 15 \\
30 & 9 & 23 \\
18 & 53 & 29
\end{array}\right)
$$

If SVD operation is useful on this matrix, then the matrix A will be decomposed into equivalent three matrices as follows: 


$$
\begin{aligned}
\mathrm{L} & =\left(\begin{array}{ccc}
-0.4019 & 0.1202 & -0.9079 \\
-0.4749 & -0.8749 & 0.9417 \\
-0.7830 & 0.4690 & 0.4083
\end{array}\right), \\
\mathrm{D} & =\left(\begin{array}{ccc}
68.5399 & 0 & 0 \\
0 & 24.2485 & 0 \\
0 & 0 & 0.5342
\end{array}\right), \\
\mathrm{U} & =\left(\begin{array}{ccc}
-0.4492 & -0.7233 & 0.5242 \\
-0.6995 & 0.6498 & 0.2972 \\
-0.5557 & -0.2332 & -0.7979
\end{array}\right)
\end{aligned}
$$

Here diagonal elements of matrix D are singular values and we observe that these values satisfy the non increasing order: $68.5399 \geq 24.2485 \geq 0.5342$.

Digital image watermarking techniques has several advantages that used singular value decomposition. Firstly, SVD transformation from the size of memory is not fixed and can be represented by a rectangle or square matrices. Secondly, SVD increase accuracy and decrease the memory requirement. Thirdly, digital images in singular values are less affected if general image watermark is executed. Fourth, singular value decomposition include by algebraic properties.

\subsection{SVD-Transformation}

We will describe a digital image watermarking technique which is based on singular value decomposition transform, such as DWT and DCT. Watermarking is established by the wavelet coefficient of selected sub bands and followed by the requirements of DCT transform on the selected sub-bands [11-14]. In this section, we will introduce the transformation of digital watermarking technique.

The DCT Transform: The discrete cosine transform is a transformation technique based on digital watermarking algorithm and spatial domain technique. The discrete cosine transform is derived from discrete Fourier transforms and represents data in terms of frequency space rather than an amplitude space. The spatial domain technique can be transformed into the frequency domain, and the frequency domain technique can be transformed back to the spatial domain by using inverse discrete cosine transform. The discrete cosine transform (DCT) is a technique for converting a signal into effortless frequency components. It represents an image as a sum of sinusoids of varying magnitudes and frequencies. With an input image, $k$ and the DCT coefficients for the transformed output image, $\mathrm{L}$ is computed according to Equation (2) as shown below. In the equation, $k$, is the input image having $\mathrm{N} \times \mathrm{M}$ pixels, $k(m, n)$ is the intensity of the pixel in row $\mathrm{m}$ and column $\mathrm{n}$ of the image and $\mathrm{L}(u, v)$ is the DCT coefficient in row $\mathrm{u}$ and column $v$ of the DCT matrix. The DCT formulas are as follows.

The general equation for a one dimension $(\mathrm{N}$ data items) DCT is defined by the following equation:

$$
\mathrm{L}(u)=\sqrt{\frac{2}{\mathrm{M}}} \beta m \sum_{x=0}^{\mathrm{M}-1} \frac{\cos (2 m+1) \pi u}{2 \mathrm{M}} k(m)
$$

and the corresponding inverse 1D DCT transform is simple $\mathrm{L}^{-1}(u)$

where

$$
\beta m=\left(\begin{array}{cc}
\frac{1}{\sqrt{2}} & u=0 \\
1 & u=1,2, \text { otherwise }
\end{array}\right.
$$

The $\beta m$ function computes the two-dimensional discrete cosine transform (DCT) of an image. The DCT has the property that, for a typical image, most of the visually significant information about the image is concentrated in just a few coefficients of the DCT. For this reason, the DCT is often used in image compression applications. The general equation for a $2 \mathrm{D}$ ( $\mathrm{N}$ by $\mathrm{M}$ image) DCT is defined by the following Equation (3):

$$
\begin{aligned}
\mathrm{L}(u, v)= & \sqrt{\frac{2}{\mathrm{M}}} \sqrt{\frac{2}{\mathrm{~N}}} \beta m \beta n \\
& \sum_{x=0}^{\mathrm{M}-1} \sum_{y=0}^{\mathrm{N}-1} \frac{\cos (2 m+1) \pi u}{2 \mathrm{M}} \frac{\cos (2 n+1) \pi u}{2 \mathrm{~N}} k(m, n)
\end{aligned}
$$

and the corresponding inverse 2D DCT transform is simple $\mathrm{L}^{-1}(u, v)$, i.e.:

where

$$
\begin{gathered}
\beta m=\left(\begin{array}{cc}
\frac{1}{\sqrt{2}} & u=0 \\
1 & u=1,2, \cdots, \mathrm{M}-1
\end{array}\right. \\
\beta n=\left(\begin{array}{cc}
\frac{1}{\sqrt{2}} & v=0 \\
1 & v=1,2, \cdots, \mathrm{N}-1
\end{array}\right.
\end{gathered}
$$

The image is reconstructed by applying inverse DCT operation according to Equation (4):

$$
\begin{aligned}
\mathrm{K}(m, n)= & \sqrt{\frac{2}{\mathrm{M}}} \sqrt{\frac{2}{\mathrm{~N}}} \beta m \beta n \\
& \sum_{u=0}^{\mathrm{M}-1} \sum_{v=0}^{\mathrm{N}-1} \frac{\cos (2 m+1) \pi u}{2 M} \frac{\cos (2 n+1) \pi u}{2 N} l(u, v)
\end{aligned}
$$

Examples for Transformation: The grayscale Lena image of $256 \times 256$ pixels, with 4-bit representation for each pixel is used as the test input. The input image is divided into 4-by-4 or 8-by-8 blocks, and the two-dimensional DCT is computed for each block. The DCT coefficients are then quantized, coded, and transmitted. 
The JPEG receiver (or JPEG file reader) decodes the quantized DCT coefficients, computes the inverse twodimensional DCT of each block, and then puts the blocks back together into a single image. For typical images, many of the DCT coefficients have values close to zero; these coefficients can be discarded without seriously affecting the quality of the reconstructed image as shown in Figure 1. The transform matrix computation method is used. The test image was compressed to different scales, from one to three and the compression ratio as well as the mean square root error of the reconstructed image were calculated for minimal error case and quantised case.

The popular block-based DCT transform segments is an image non-overlapping blocks and applies DCT to each block. This result in giving three frequency coefficient sets: low frequency sub-band, mid frequency subband, and high frequency sub band. The digital watermarking based on two facts. The first fact is that much of the signal energy lies at low- frequency sub-band which includes the most important visual parts of the image. The second fact is that high frequency components of the image are generally detached through compression and noise attacks. The watermark is surrounded by modifying the coefficients of the middle frequency sub-band so that the visibility of the image will not be overstated and the watermark will not be removed by compression.

Discrete Wavelet Transform: The transformation product is a set of coefficients organized in the way that enables not only spectrum analyses of the signal, but also spectral behavior of the signal in time. This is achieved by decomposing signal, breaking it into two components, each concerned information about source signal. Filters from the filter bank used for decomposition come in pairs: low pass and high pass. Low pass filtered signal contains information about slow changing component of the signal, looking very similar to the original signal, only two times shorter in term of number of samples. High pass filtered signal be full of information about fast changing component of the signal. In most cases, high pass component is not so rich with data offering good property for compression. In some cases, such as audio or video signal, it is possible to contend with some of the samples of the high pass component without noticing any significant changes in signal. Filters from the filter bank are called wavelets and as shown in Figure 2.

For 2-D images, applying DWT corresponds to processing the image by 2-D filters in each dimension. The filters divide the input image into four non-overlapping multi-resolution sub-bands $\mathrm{LL}_{1}, \mathrm{LH}_{1}, \mathrm{HL}_{1}$ and $\mathrm{HH}_{1}$. The sub-band $\mathrm{LL}_{1}$ represents the coarse-scale DWT coefficients while the sub-bands $\mathrm{LH}_{1}, \mathrm{HL}_{1}$ and $\mathrm{HH}_{1}$ represent the fine-scale of DWT coefficients. To obtain the next coarser scale of wavelet coefficients, the sub-band $\mathrm{LL}_{1}$ is further processed until some final scale $\mathrm{N}$ is reached. When $\mathrm{N}$ is reached we will have $3 \mathrm{~N}+1$ sub-bands consisting of the multi-resolution sub-bands $\mathrm{LL}_{\mathrm{y}}$ and $\mathrm{LH}_{\mathrm{y}}$, $\mathrm{HL}_{\mathrm{y}}$ and $\mathrm{HH}_{\mathrm{y}}$ where y ranges from 1 until N. It has the following steps in digital image watermarking transformation such as

Step 1: Present DWT on the original image to decompose it into four non-overlapping multi-resolution coefficient sets, such as $\mathrm{LL}_{1}, \mathrm{HL}_{1}, \mathrm{LH}_{1}$ and $\mathrm{HH}_{1}$.

Step 2: Present DWT again on two $\mathrm{HL}_{1}$ and $\mathrm{LH}_{1}$ subbands to get eight smaller sub-bands and prefer four coefficient sets: $\mathrm{HL}_{12}, \mathrm{LH}_{12}, \mathrm{HL}_{22}$ and $\mathrm{LH}_{22}$ as shown in Figure 1.
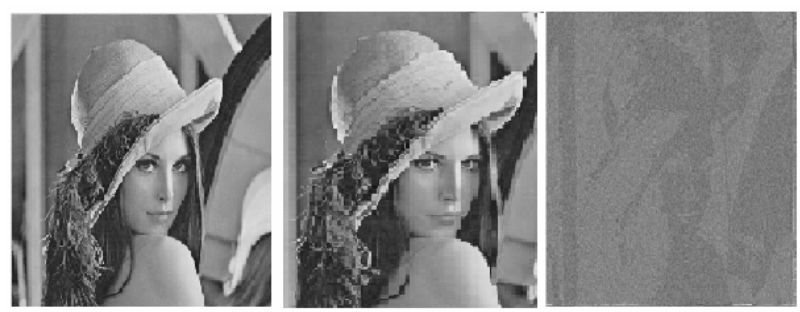

Figure 1. Reconstructed image, compression image and quantization image by using DCT.

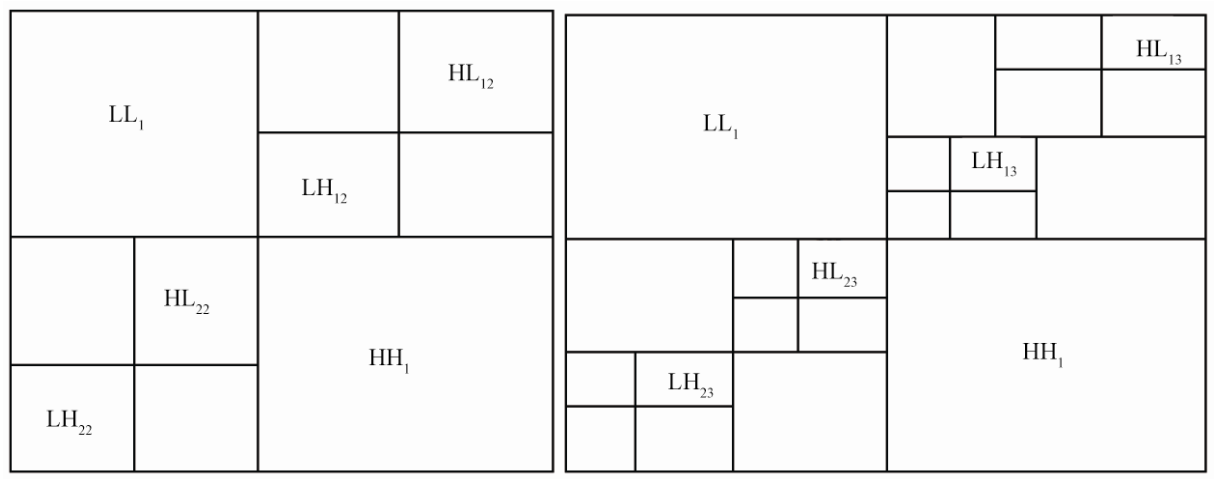

Figure 2. Sketch map of DWT and DCT decomposed sub-bands. 
Step 3: Present DWT again on four sub-bands, such as $\mathrm{HL}_{12}, \mathrm{LH}_{12}, \mathrm{HL}_{22}$ and $\mathrm{LH}_{22}$ to get sixteen smaller coefficient sets and prefer four coefficient sets, such as $\mathrm{HL}_{13}$, $\mathrm{LH}_{13}, \mathrm{HL}_{13}$ and $\mathrm{LH}_{13}$ as shown in Figure 1.

Step 4: Divide four coefficient sets such as $\mathrm{HL}_{13}, \mathrm{LH}_{13}$, $\mathrm{HL}_{23}$ and $\mathrm{LH}_{23}$ into $4 \times 4$ blocks.

Step 5: Present DCT to each block in the chosen subbands $\left(\mathrm{HL}_{13}, \mathrm{LH}_{13}, \mathrm{HL}_{23}\right.$ and $\left.\mathrm{LH}_{23}\right)$. These coefficient sets are chosen to inquire both of imperceptibility and strength of algorithm equally.

The DWT is very suitable to identify the areas in the original image where a watermark can be embedded effectively. This property allows the utilization of the masking effect of the human visual system such that if a DWT coefficient is modified, only the region corresponding to that coefficient will be modified. In general most of the image energy is concentrated at the lower frequency sub-bands LLx and therefore embedding watermarks in these sub-bands may humiliate the image appreciably. Embedding in the low frequency sub-bands, however, could increase robustness appreciably. On the other hand, the high frequency sub-bands HHx include the edges and textures of the image and the human eye is not generally sensitive to changes in such sub-bands. This allows the watermark to be embedded without being superficial by the human eye. The compromise accepted by many DWT-based watermarking algorithm, is to embed the watermark in the middle frequency sub-bands LHy and HLy where good enough performance of imperceptibility and robustness could be achieved.

\section{Proposed Watermarking Techniques}

We proposed a singular value decomposition technique and quantization based watermarking technique. The watermarking techniques can be represented by three components, L, D and U. It relies on row and column operations. Row operations involve pre-multiplying matrix and column operations involve post-multiplying matrix. The D component can be explored with a diagonal matrix. These techniques depend upon the watermark embedding algorithm and watermark extracting algorithm.

\subsection{Watermark Embedding Algorithm}

The digital image watermarking algorithm can be followed by singular value decomposition techniques, which involve the characteristics of the $\mathrm{D}$ and $\mathrm{U}$ components. In the embedding algorithm, the largest coefficients in D component were customized and used to embed a watermark. The adaptation was determined by the quantization method. We will start the algorithm by applying
SVD transformation on original image and to reconstruct the watermarked image. Because the largest coefficients in the $\mathrm{D}$ component can oppose with general image processing, the embedded watermark was not really affected. In this way, the quality of the watermarked image can be decomposed by quantization method. In our inspection, two important features of the D and U components are found. In the first feature, the number of non zero coefficients in the $\mathrm{D}$ component could be used to determine the complexity of a matrix. Commonly, the greater number of the non-zero coefficient can be specified by greater complexity. In the second feature, the relationship between the coefficients in the first column of the L component could be sealed, when usually image processing was presented as shown in Figure 3. The watermarks embedding algorithm can be described as follows.

Step 1: Read the original host image.

Step 2: Partition the image into blocks of $n \times n$ pixels.

Examples: Perform combination of two filters as prefiltering operation. The first filter is $3 \times 3$ sharpening filter which is defined as Equation (5).

$$
\left(\begin{array}{ccc}
0 & -1 & 0 \\
-1 & 3 & -1 \\
0 & -1 & 0
\end{array}\right)
$$

This filter enhances contrast of watermarked image. The second filter is designed by Laplacian of Gaussian filter and defined by general equation as 6 .

$$
\mathrm{G}(x)=\frac{1}{\sqrt{2 \pi \sigma^{2}}} \mathrm{e}^{-\frac{x^{2}}{2 \sigma^{2}}}
$$

The Gaussian blur is types of image-blurring filter that uses a Gaussian function (which also expresses the normal distribution in statistics) for calculating the transformation to apply to each pixel in the image as shown by Equation (7).

$$
\mathrm{G}(x, y)=\frac{1}{2 \pi \sigma^{2}} \mathrm{e}^{-\frac{x^{2}+y^{2}}{2 \sigma^{2}}}
$$

where $x$ is the distance from the origin in the horizontal axis, $y$ is the distance from the origin in the vertical axis, and $\sigma$ is the standard deviation of the Gaussian distribution. The default value for them in $g=4$ and $\sigma=0.5$.

Performing these two filters on watermarked image could caused details of image become more visible, its means that watermark information which is different from image background become recognizable uncomplicatedly.

Step 3: Perform singular value decomposition (SVD) transformation. 
Step 4: Extract the greater coefficient $\mathrm{D}(1,1)$ from each $\mathrm{D}$ component and quantize by using a predefined quantization coefficients A. Suppose that $S=D(1,1)$ $\bmod$ A.

Step 5: Perform embed the two pseudo-random sequences PN0, PN1, that is applied to the mid-band coefficients. If $A$ is the matrix of the mid band coefficients of SVD transformed block, then embedding is done as follows:

If the watermark bit is 0 then,

$$
\begin{gathered}
\mathrm{D}^{\prime}(1,1)=\mathrm{D}(1,1)+\mathrm{K} / 4-\mathrm{A} \text {, so that }[\mathrm{A}<3 \mathrm{~K} / 4] \\
\text { Otherwise, if the watermark bit is } 1 \text { then, } \\
\mathrm{D}^{\prime}(1,1)=\mathrm{D}(1,1)-\mathrm{K} / 4+\mathrm{A} \text {, so that }[\mathrm{A}<\mathrm{K} / 4]
\end{gathered}
$$

Step 6: Perform the inverse of singular value decomposition transformation to reform the watermarked image.

\subsection{Watermark Extracting Algorithm}

The watermark extracting algorithm is similar to the watermark embedding algorithm. Extraction algorithm is the same as embedding and pre-filtering is used before applying SVD transform to superior split watermark information from original image. The watermark extraction algorithm is performed as described by the following steps. The first three steps of the watermark extracting algorithms are same as the watermark embedding algorithm except that the original image is replaced with the watermarked image. Previously, an embedded block is detected according to the feature of the $\mathrm{D}$ component and PRNG, the relationship of the U component coefficients is observed. If a positive relationship is detected, the extracted watermark has assigned a bit value of 1 . Otherwise, the extracted watermark has assigned a bit value of 0 . These extracted bit values convert the original image SVD from the extracted watermark. The extracted watermark can be specified by original watermarked image and as shown in Figure 4.

Step 1: Read the watermarked image.

Step 2: watermarked it into blocks of $n \times n$ pixels.

Step 3: Perform the SVD transformation.

Step 4: Extract the greater coefficients D" $(1,1)$ from each $\mathrm{D}$ component and quantize by using a predefined quantization coefficients $A$. Suppose that $S=D^{\prime}(1,1)$ $\bmod$ A.

Step 5: Regenerate the two pseudo random sequences number using the same key, which is used in the watermark embedding algorithm.

Step 6: For an extraction watermark bit valued of zero, if $A<K / 2$. On the other hand, the extraction watermark bit value of one, if $\mathrm{A}>\mathrm{K} / 2$.

Step 7: The watermark is restructured using the ex-

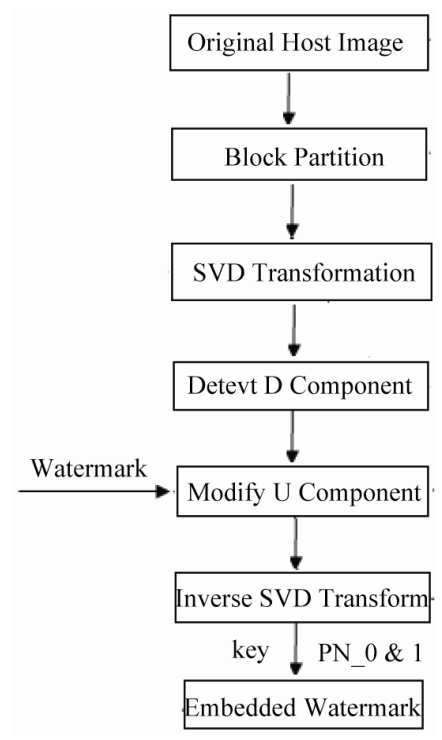

Figure 3. Flow chart for watermark embedding algorithm.

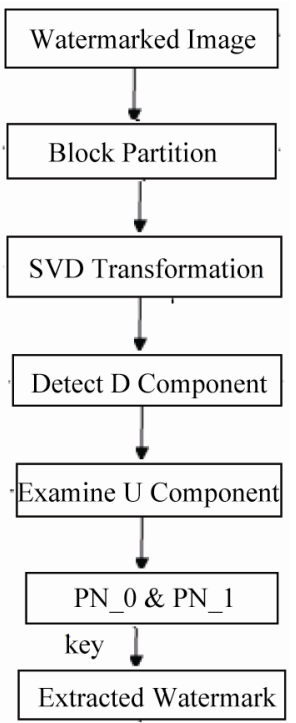

Figure 4. Flow chart for watermark embedding algorithm.

tracted watermark bits, and compute the similarity between the original watermark and extracted watermarks.

\section{Performance Evaluation}

We evaluated the performance of the SVD image watermarking algorithms. The performance of the watermarking methods can be measured by imperceptibility and robust capabilities. Imperceptibility means that the superficial quality of the original image should not be distorted by the presence of watermark image and as shown by Equation (8). On the other hand, the robustness is a measure of the intentionally attacks and unintention- 
ally attacks. It was found that the image quality measured by peak signal to noise ratio among the watermarked images was larger than $42 \mathrm{db}$ [15]. This peak signal to noise ratio is defined as

$$
\operatorname{PSNR}\left(o, o^{1}\right)=10 \log _{10}\left(\frac{255 \times 255}{\sum_{i=1}^{m} \sum_{j=1}^{n} \frac{1}{x y}\left(o-o^{1}\right)^{2}}\right)
$$

The PSNR is employed to evaluate the difference between an original image $o$ and watermarked image $o^{1}$. For the robust capability, mean absolute error (MSE) measures the difference between an original watermark $\mathrm{W}$ and corresponding extracted watermark $\mathrm{W}^{1}$ as shown by Equation (9).

$$
\operatorname{MSE}\left(w^{0}, w^{1}\right)=\sum_{i=0}^{d}\left(\frac{w^{0}-w^{1}}{w}\right)
$$

Generally, if PSNR value is larger than $40 \mathrm{db}$ the watermarked image is within acceptable degradation levels, i.e. the watermarked is almost invisible to human visual system. A lower mean absolute error reveals that the extracted watermark $w^{0}$ resembles the $w^{1}$ more closely. The strength of digital watermarking method is accessed from the watermarked image $o^{1}$, which is further degraded by attacks and the digital watermarking performance of proposed method is compared with that of Chen [4]. If a method has a lower $\operatorname{MSE}\left(w^{0}, w^{1}\right)$, it is more robust.

\section{Experimental Results}

The experimental results are simulated with the software MATLAB 7.10 version. It provides a single platform for computation, visualization, programming and software development. All problems and solutions in Matlab are expressed in notation used in linear algebra and essentially involve operations using matrices and vectors. We are using a $256 \times 256$ "Lena", "facial", and "Moon" as the gray scale original host image, and a $256 \times 256$ greyscale image of the watermark image. The three images are shown in Figures 5 and $\mathbf{6}$ respectively. In the proposed method, we select the largest complexity of blocks; the original images can be separated into blocks of $4 \times 4$ pixels. Each block can be transformed into L, D, and $\mathrm{U}$ components by singular value decomposition. And then, a set of blocks with the same size as the watermark was selected, according to the feature of the D component. For an embedding watermark block, the relationship between the L component coefficients can be examined and the coefficients were modified, according to the watermark to be embedded. In our experiment, the original image and watermarked image quality is shown in Figure 5.

We claimed the embedding algorithm and extracting algorithm to identify the ownership of the original watermarked image as shown in Tables $\mathbf{1}$ and $\mathbf{2}$. We can see that the performance of our algorithm against the different attacks. Further, the proposed watermarking algorithm can be used for protecting the copyright of digital images. It can be observed from Tables 1 and 2 that the future method provides excellent results in the geometrical attacks.

Simulation results suggest that this digital watermarking algorithm is robust against many common different types of attacks such as cropping attacks, pyramid at-

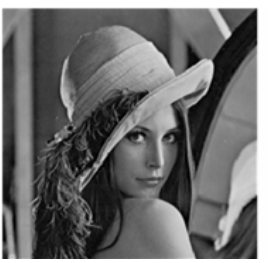

(a)

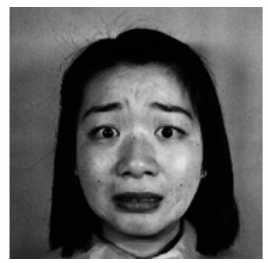

(b)

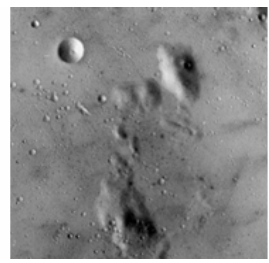

(c)
Figure 5. Three original images of $256 \times 256$ pixels (a) The original lena image (b) The original facial image (c) The original moon image.

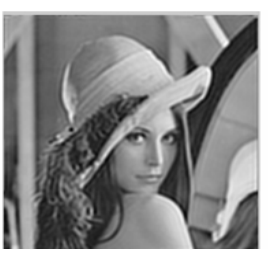

(a)

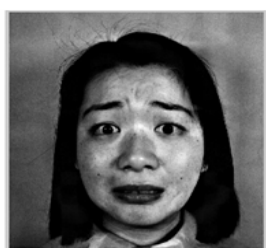

(b)

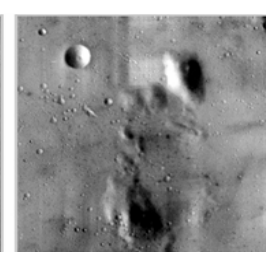

(c)
Figure 6. Three watermarked images of $256 \times 256$ pixels (a) The watermarked lena image; (b) The watermarked facial image; (c) The watermarked moon image.

\begin{tabular}{|c|c|c|c|c|c|c|c|}
\hline Images Type & Without Attacks & Cropping Attacks & Pyramid Attacks & Rotation Attacks & Noise Attacks & Blurring Attacks & PSNR (DB) \\
\hline Lena Image & 29.2752 & 38.7569 & 45.3102 & 30.0444 & 26.8879 & 29.2752 & $\alpha=0.2$ \\
\hline Facial Image & 29.9337 & 35.2695 & 46.3784 & 31.3159 & 26.5601 & 29.9337 & $\alpha=0.2$ \\
\hline Moon Image & 24.0375 & 36.3759 & 42.8253 & 26.2853 & 24.0375 & 24.0375 & $\alpha=0.2$ \\
\hline
\end{tabular}

Table 1. The experimental results of the error ratio of the embedded watermark after different attacks by proposed method. 
Table 2. The experimental results of the error ratio of the extracted watermark after different attacks by the proposed method.

\begin{tabular}{cccccccc}
\hline Images Type & Without Attacks & Cropping Attacks & Pyramid Attacks & Rotation Attacks & Noise Attacks & Blurring Attacks & PSNR (DB) \\
\hline Lena Image & 272.2211 & 279.3277 & 282.3816 & 273.8656 & 283.2165 & 272.2217 & $\alpha=0.2$ \\
Facial Image & 284.1641 & 283.9997 & 280.3740 & 285.8853 & 279.7679 & 284.1641 & $\alpha=0.2$ \\
Moon Image & 275.1305 & 271.2818 & 287.8321 & 278.3293 & 275.1305 & 275.1305 & $\alpha=0.2$ \\
\hline
\end{tabular}

tacks, rotation attacks, and noise attacks and blurring attacks Figures 7-12. However, cropping is a geometrical manipulation and rotation is a geometrical distortion in practical application. If alpha's value is more than 0.2 then quality of original image and watermarked image is not good. So we are using the dumpy value in these techniques.

We are using the different coefficent of parameters by proposed method based on digital image watermarking embedding algorithm and extracting algorithm as a shown by Tables 3 and 4 . Its depends upon the security analysis and as shown in Figures 13-30. The parameters are to be satisfying by E1, E2 and E3.

E1 is the parameter of "Lena Image", E2 is the parameter of "Facial Image" and E3 is the parameter of "Moon Image".
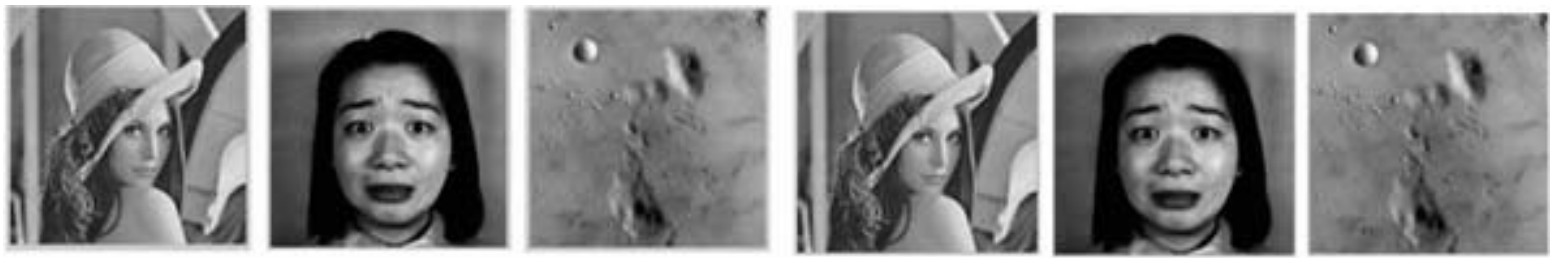

Figure 7. Grey level of original image and watermarked image under without attacks and the corresponding qualities: (a) Lena (29.2752 db), (272.2211 db), (b) Facial (29.9337 db), (284.1641 db), (c) Moon (24.0375 db), (275.1305 db).
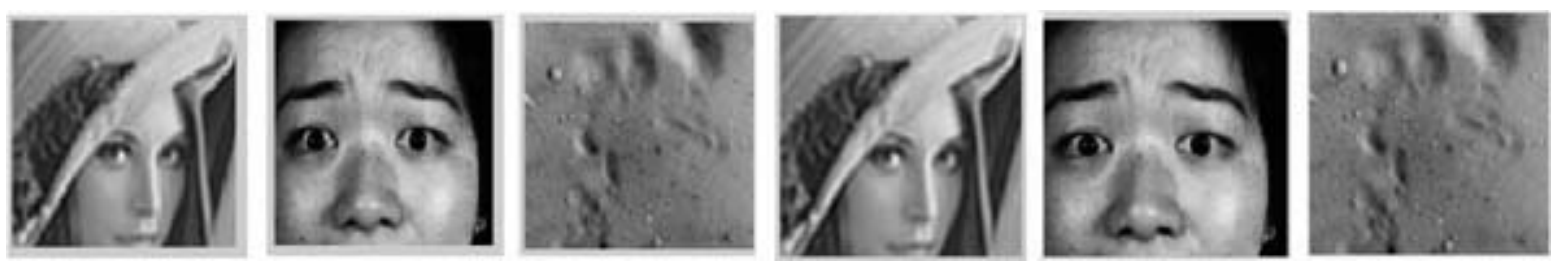

Figure 8. Grey level of original image and watermarked image under cropping attacks and the corresponding qualities: (a) Lena (38.7569 db), (279.3277 db), (b) Facial (35.2695 db), (283.9997 db), (c) Moon (36.3759 db), (271.2718 db).
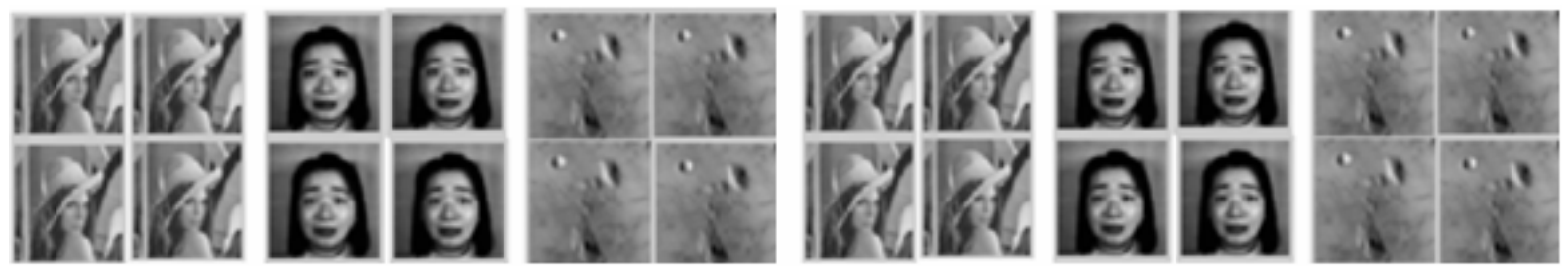

Figure 9. Grey level of original image and watermarked image under pyramid attacks and the corresponding qualities: (a) Lena (45.3102 db), (288.3216 db), (b) Facial (46.3784 db), (280.3740 db) (c) Moon (42.8253 db), (287.8721 db).
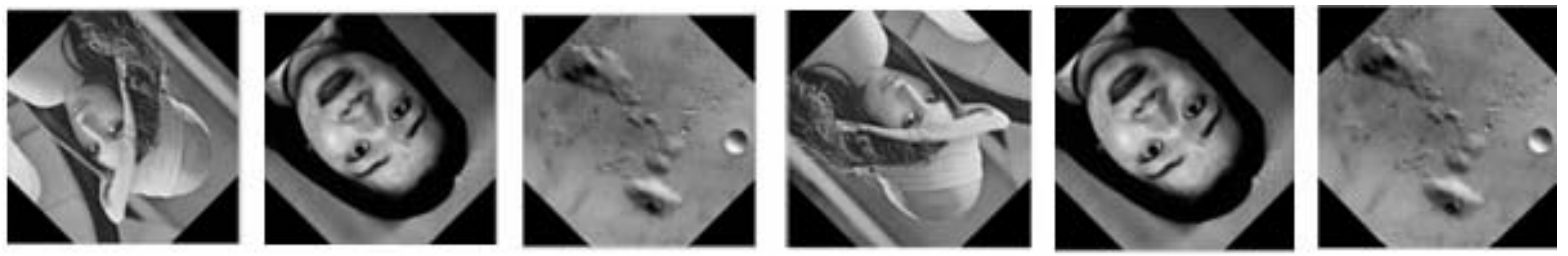

Figure 10. Grey level of original image and wtaremarked image under rotation attacks and the corresponding qualities: (a) Lena (30.0444 db), 273.8656 (db), (b) Facial (31.3159 db), 285.8853 (db) (c) Moon (26.2853 db), 278.3293(db). 

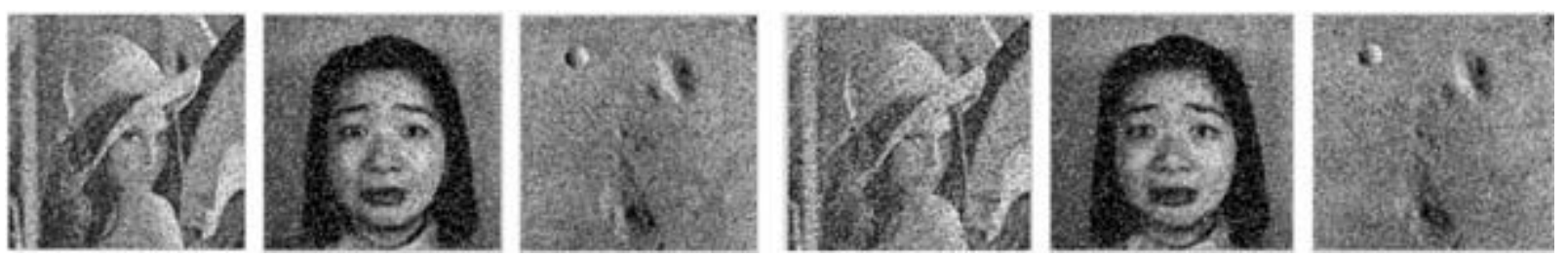

Figure 11. Grey level of original image and watermarked image under noise attacks and the corresponding qualities: (a) Lena (26.8879 db), 283.2165 (db) (b) Facial (26.5601 db), 279.7678 (db) (c) Moon (24.0375 db), 275.1305 (db).
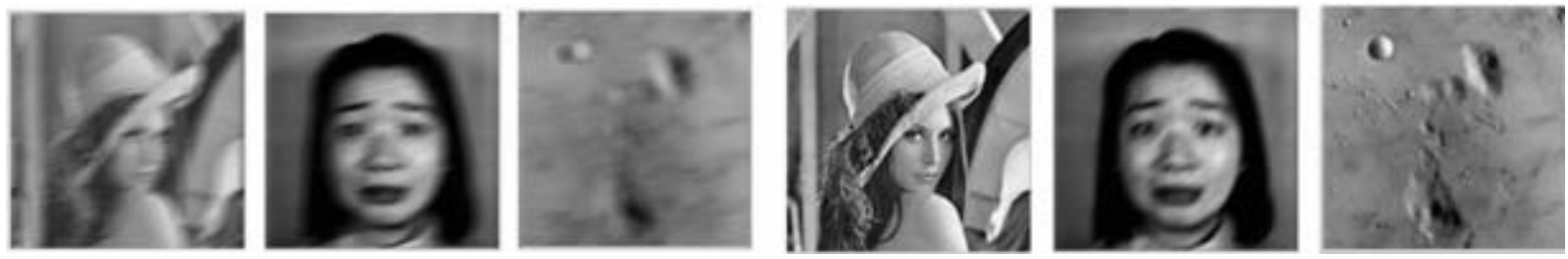

Figure 12. Grey level of original image and watermarked image under blurred attacks and the corresponding qualities: (a) Lena (26.8879 db), 272.2217 (db), (b) Facial (26.5601 db), 284.1641 (db), (c) Moon (24.0375 db), 275.1305 (db).

Table 3. A similarity between coefficients of original image and watermarked image using different parameters by proposed method under embedded algorithm.

\begin{tabular}{cccccccc}
\hline Coefficent of Parameters Without Attacks & Cropping Attacks Pyramid Attacks & Rotation Attacks & Noise Attacks & Blurring Attacks & PSNR (DB) \\
\hline E1 & 29.2753 & 38.7691 & 29.2752 & 30.4487 & 27.4361 & 23.5507 & $\alpha=0.2$ \\
E2 & 29.9334 & 35.3072 & 38.4104 & 10.0200 & 27.5376 & 29.9347 & $\alpha=0.2$ \\
E3 & 24.0375 & 36.3290 & 24.0375 & 11.1151 & 12.4233 & 23.5507 & $\alpha=0.2$ \\
\hline
\end{tabular}

Table 4. A similarity between coefficients of original image and watermarked image using different parameters by proposed method under extracted algorithm.

\begin{tabular}{cccccccc}
\hline Coefficent of Parameters Without Attacks & Cropping Attacks & Pyramid Attacks & Rotation Attacks & Noise Attacks & Blurring Attacks & PSNR (DB) \\
\hline E1 & 272.2212 & 279.3657 & 272.2212 & 273.8651 & 271.7452 & 272.4247 & $\alpha=0.2$ \\
E2 & 241.1641 & 285.0083 & 278.8258 & 284.1675 & 284.8783 & 284.1667 & $\alpha=0.2$ \\
E3 & 275.1305 & 291.1681 & 275.5309 & 275.1345 & 273.5305 & 276.7801 & $\alpha=0.2$ \\
\hline
\end{tabular}
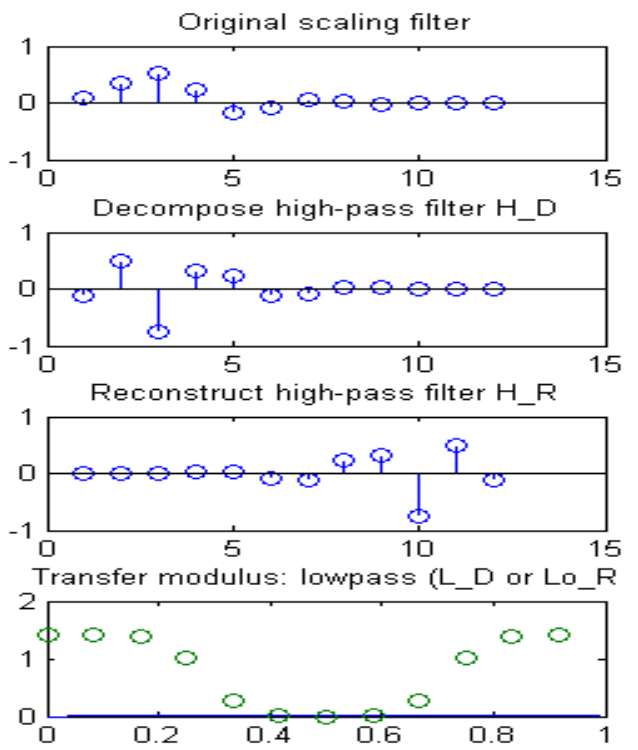
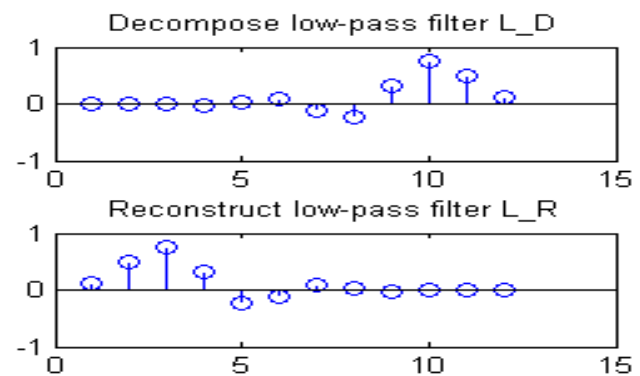

The Original and watermarked Lena without Image

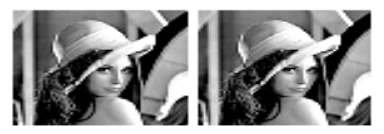

Figure 13. A similarity between coefficents of original watermarked image under without attacks: (a) Lena without attacks (29.2753 db), (272.2212 db). 

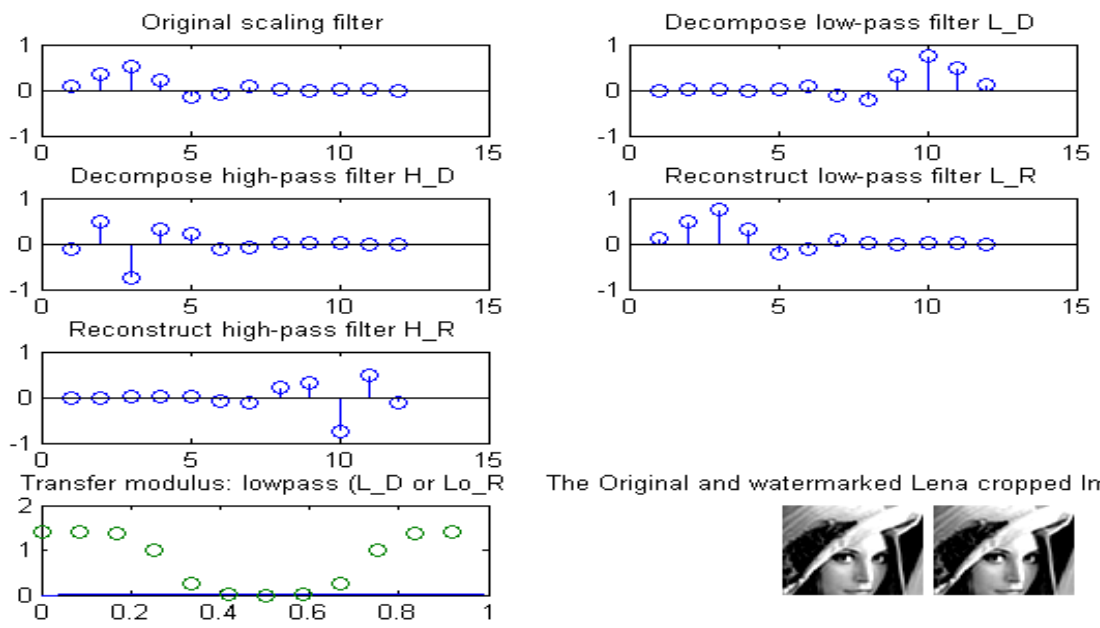

The Original and watermarked Lena cropped Image

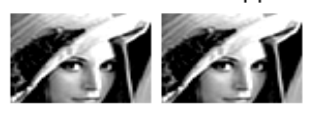

Figure 14. A similarity between coefficents of original watermarked image under cropping attacks: (a) Lena cropped attacks (38.7691 db, 279.3657db).
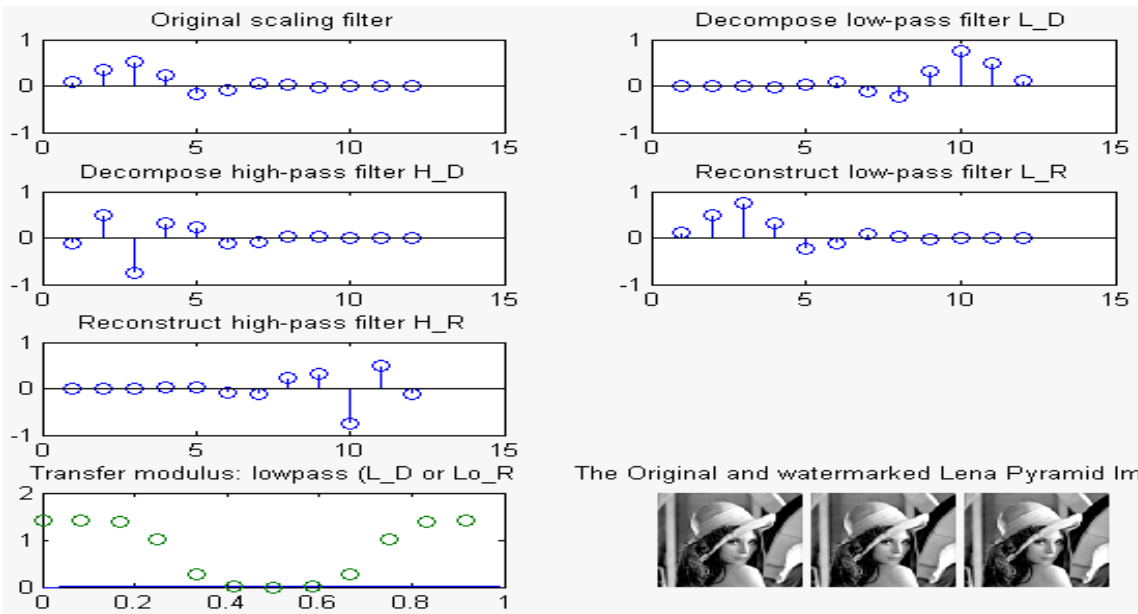

The Original and watermarked Lena Pyramid Image

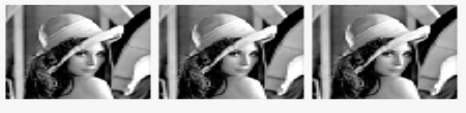

Figure 15. A similarity between coefficents of original watermarked image under pyramid attacks: (a) Lena pyramid attacks (29.2752), (272.2212 db).
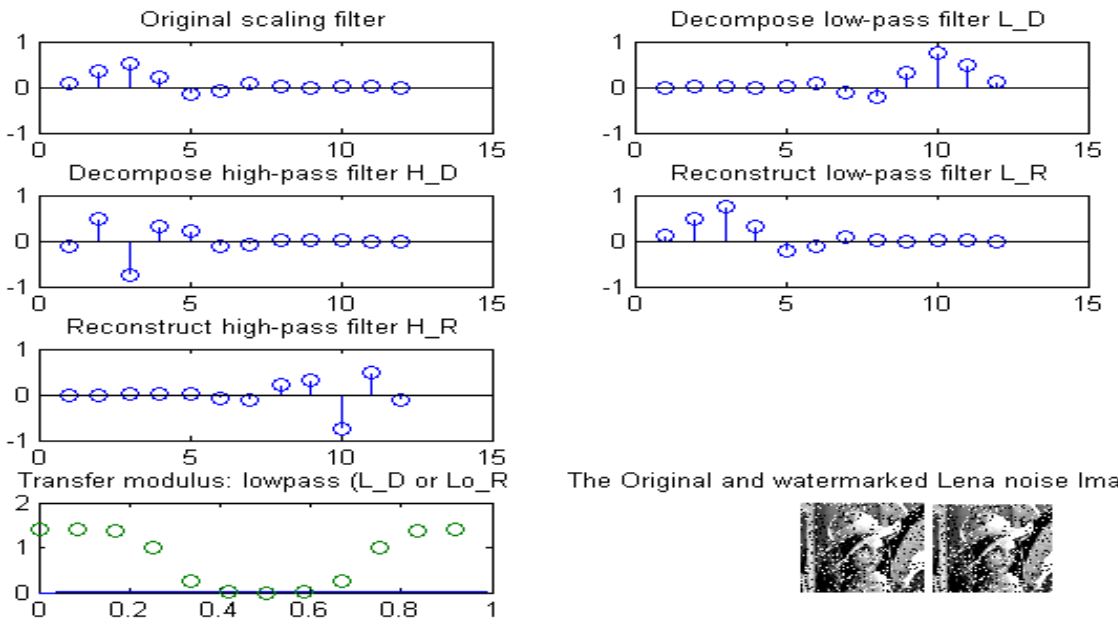

The Original and watermarked Lena noise Image

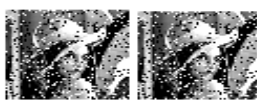

Figure 16. A similarity between coefficents of original watermarked image under noise attacks: (a) Noise attacks (27.4361 db), (271.7452 db). 

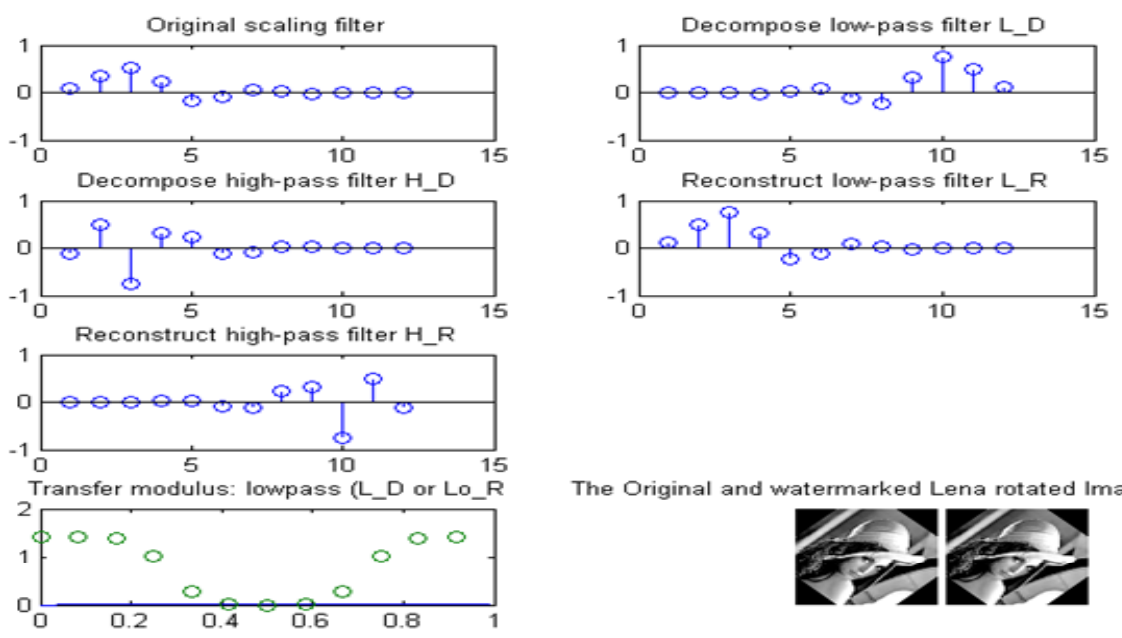

The Original and watermarked Lena rotated Image

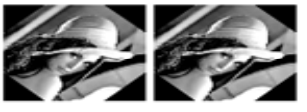

Fgure 17. A similarity between coefficents of original watermarked image under rotated attacks: (a) Lena rotated attacks (30.4487 db), (273.865 db).
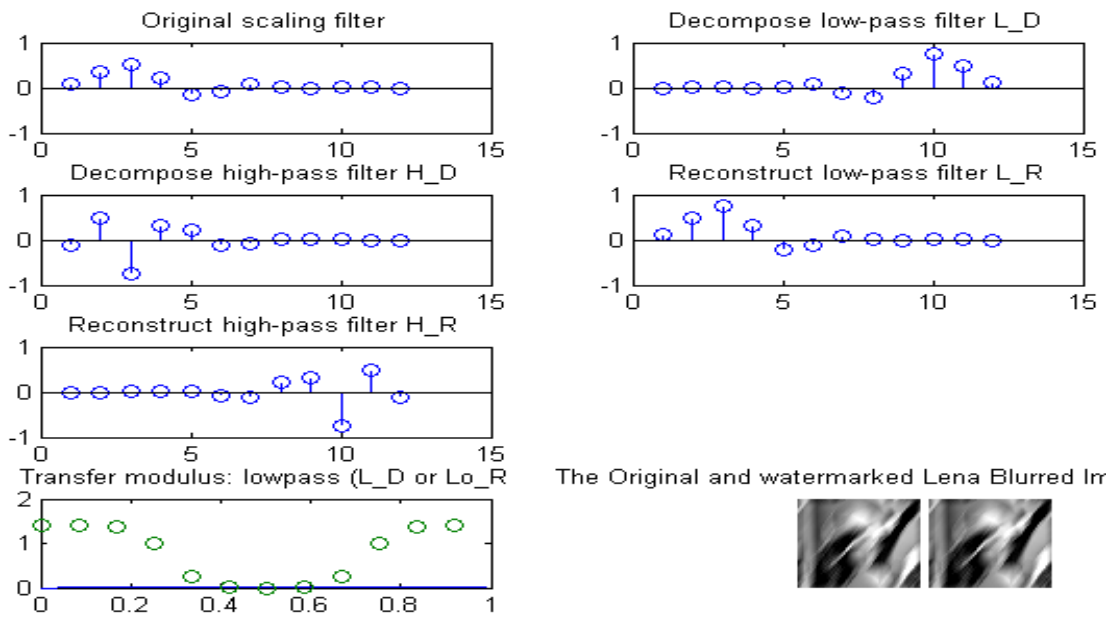

The Original and watermarked Lena Blurred Image

Figure 18. A similarity between coefficents of original watermarked image under blurring attacks: (a) Lena blurring attacks (23.5507 db), (272.4247 db).
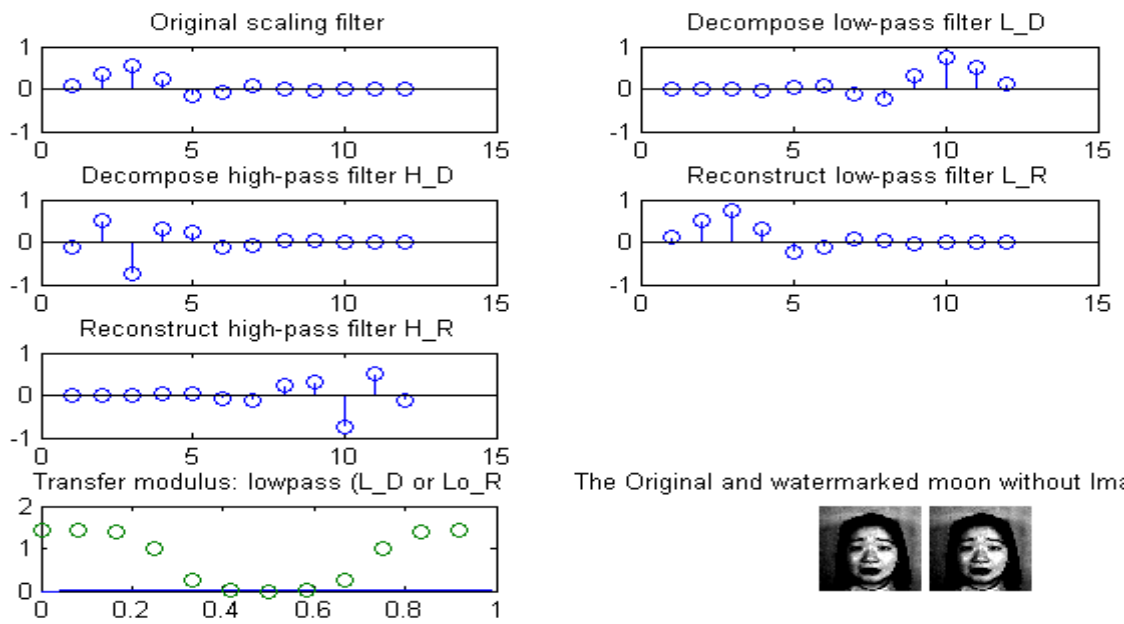

The Original and watermarked moon without Image

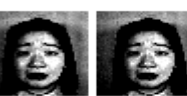

Figure 19. A similarity between coefficents of original watermarked image under without attacks: (a) Facial without attacks (29.9337 db), (284.1641 db). 

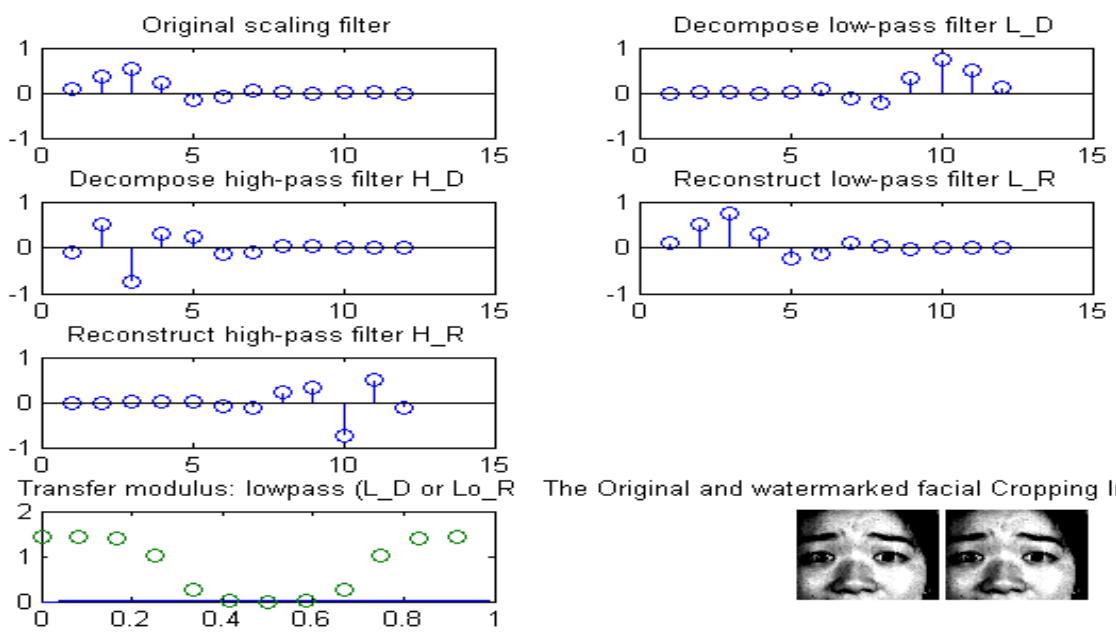

The Original and watermarked facial Cropping Image

Figure 20. A similarity betwee
(35.3072 db), (285.0083 db).
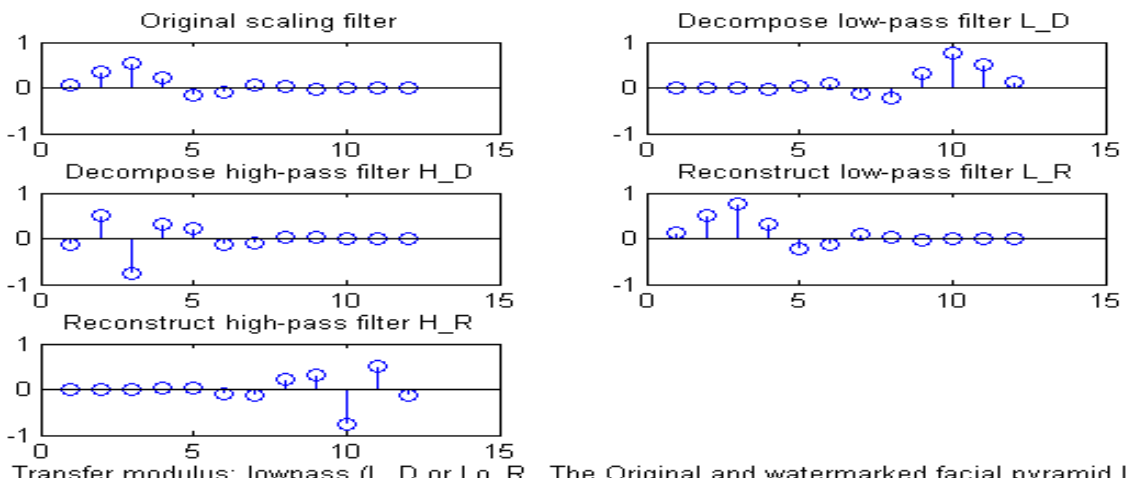

Transfer modulus: lowpass (L_D or Lo_R The Original and watermarked facial pyramid Image
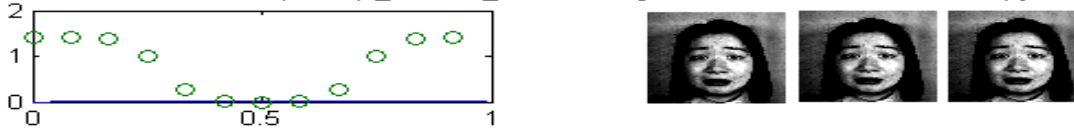

Figure 21. A similarity between coefficents of original watermarked image under pyramid attacks: (a) Facial pyramid attacks (38.4104 db), (278.8258 db).
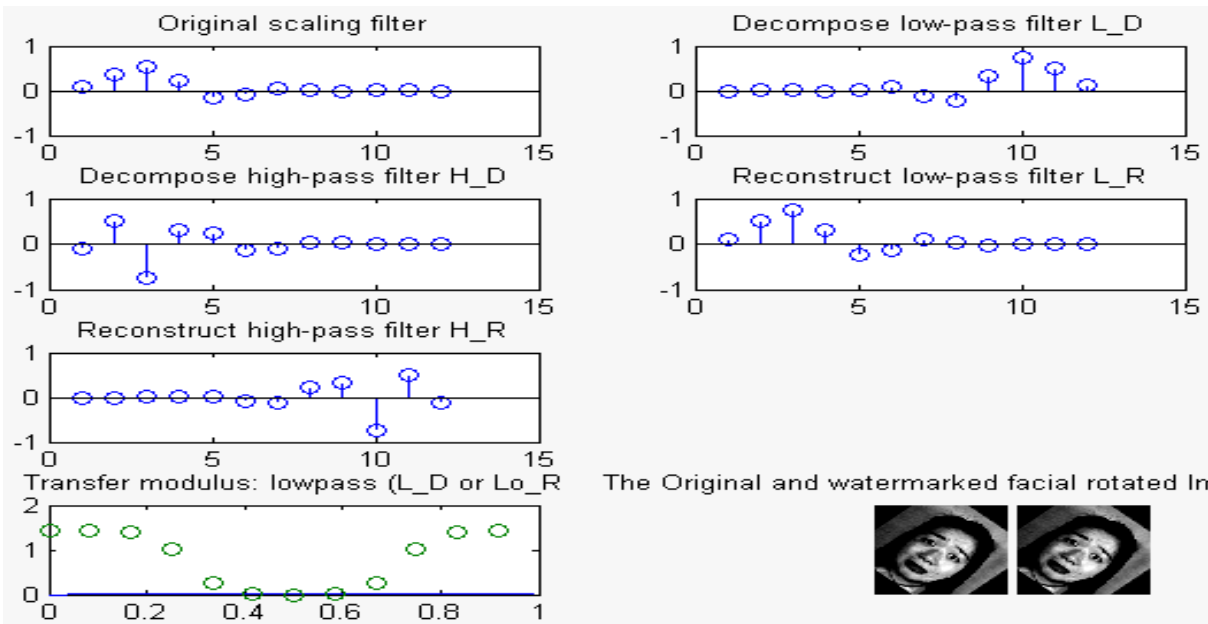

The Original and watermarked facial rotated Image

Figure 22. A similarity between coefficents of original watermarked image under rotated attacks: (a) Facial rotated attacks (10.0200 db), (284.1675 db). 

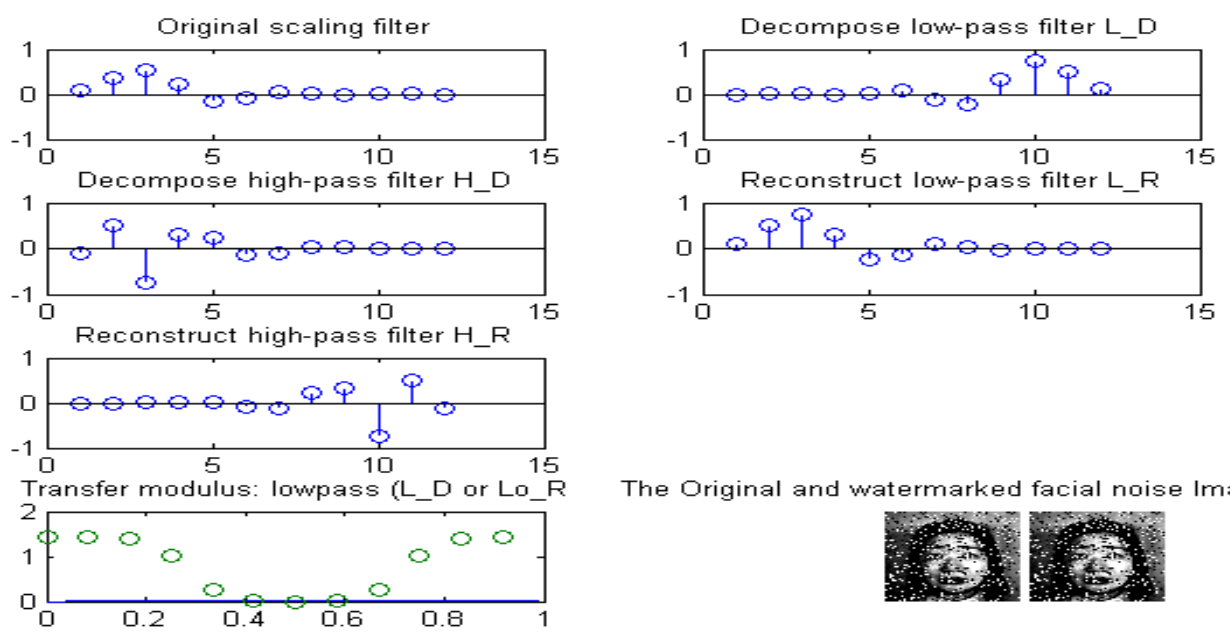

The Original and watermarked facial noise Image

Figure 23. A similarity between coefficents of original watermarked image under noise attacks: (a) Facial noise attacks (27.5376 db), (284.8483 db).
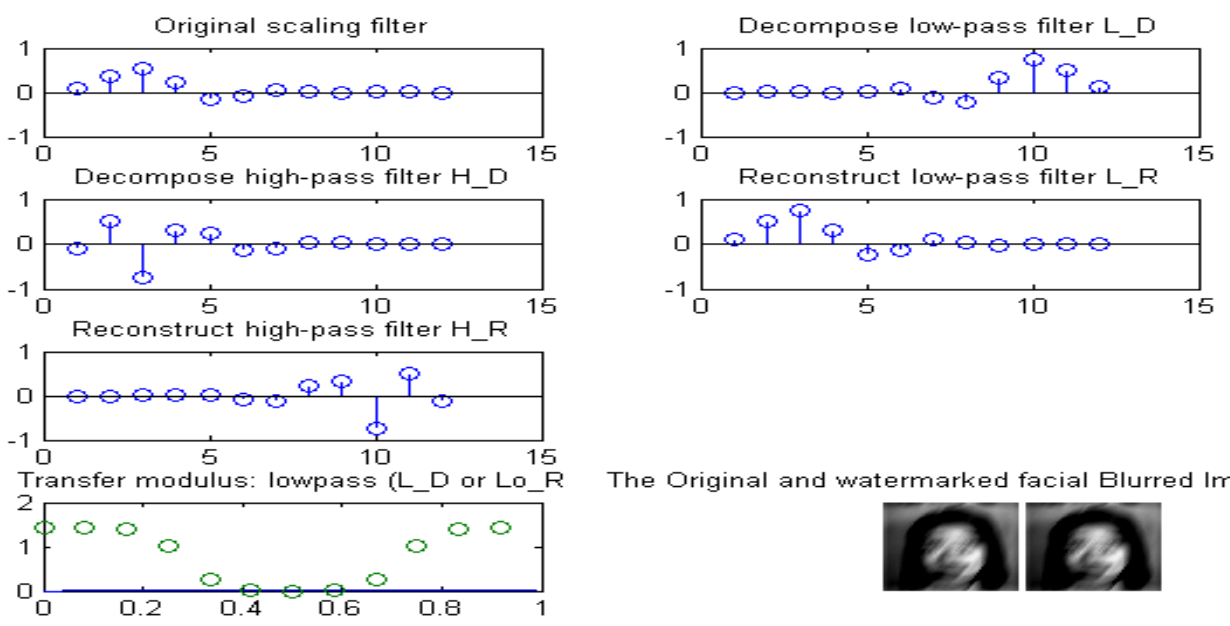

The Original and watermarked facial Blurred Image

Figure 24. A similarity between coefficents of original watermarked image under blurring attacks: (a) Facial blurred attacks (29.9347 db), (284.1667 db).
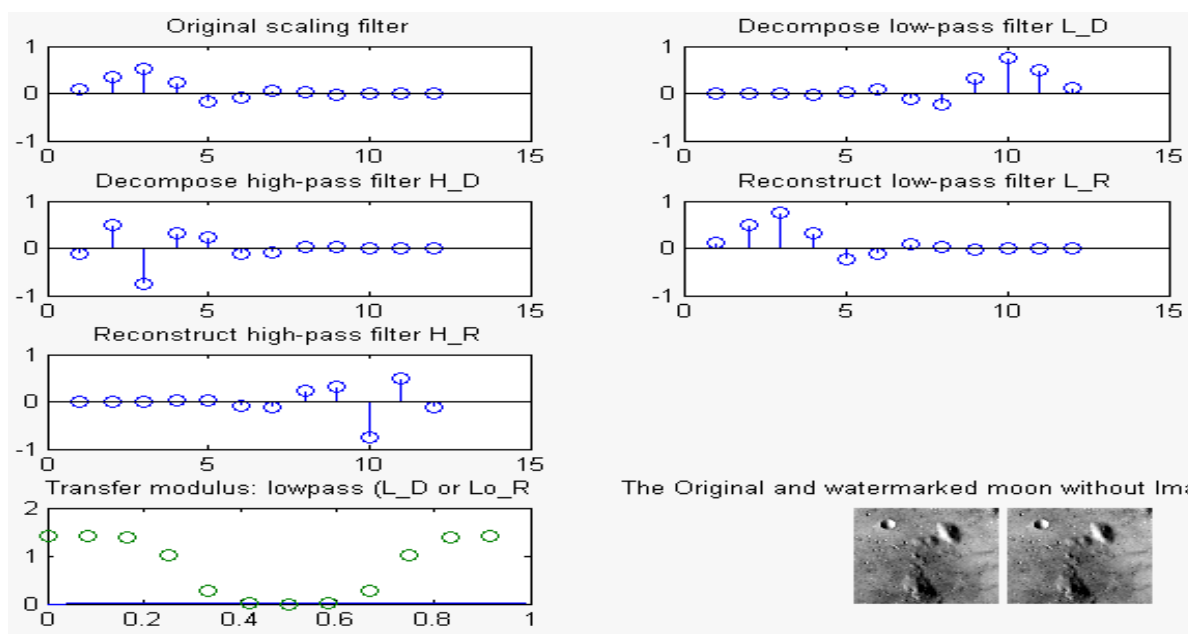

The Original and watermarked moon without Image

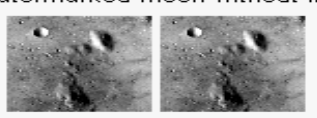

Figure 25. A similarity between coefficents of original watermarked image under without attacks (a) Moon without attacks (24.0375 db), (275.1305 db). 

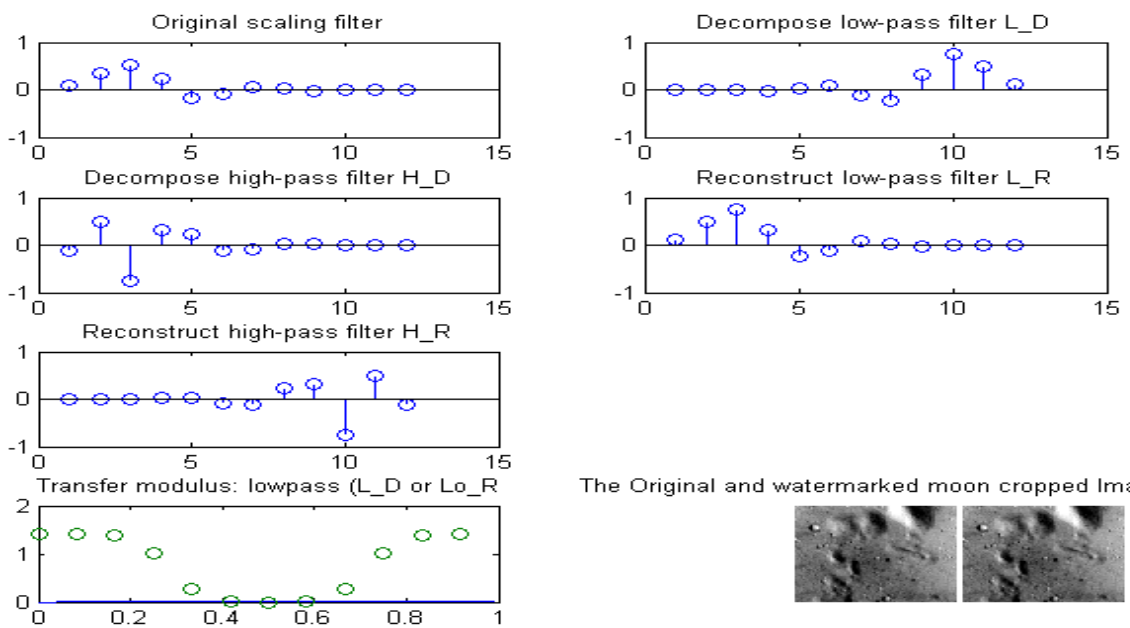

The Original and watermarked moon cropped Image

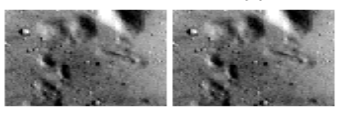

Figure 26. A similarity between coefficents of original watermarked image under cropped attacks: (a) Moon cropped attacks (36.329 db), (291.1681 db).
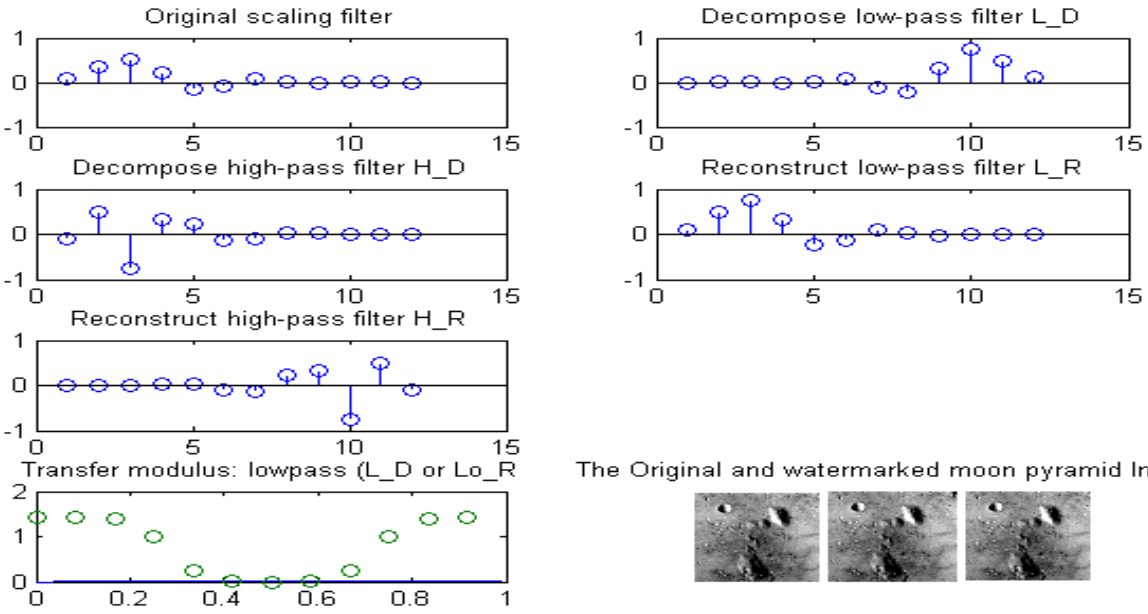

The Original and watermarked moon pyramid Image

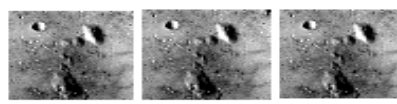

Figure 27. A similarity between coefficents of original watermarked image under pyramid attacks: (a) Moon pyramid attacks (24.0375 db), (275.5309 db).
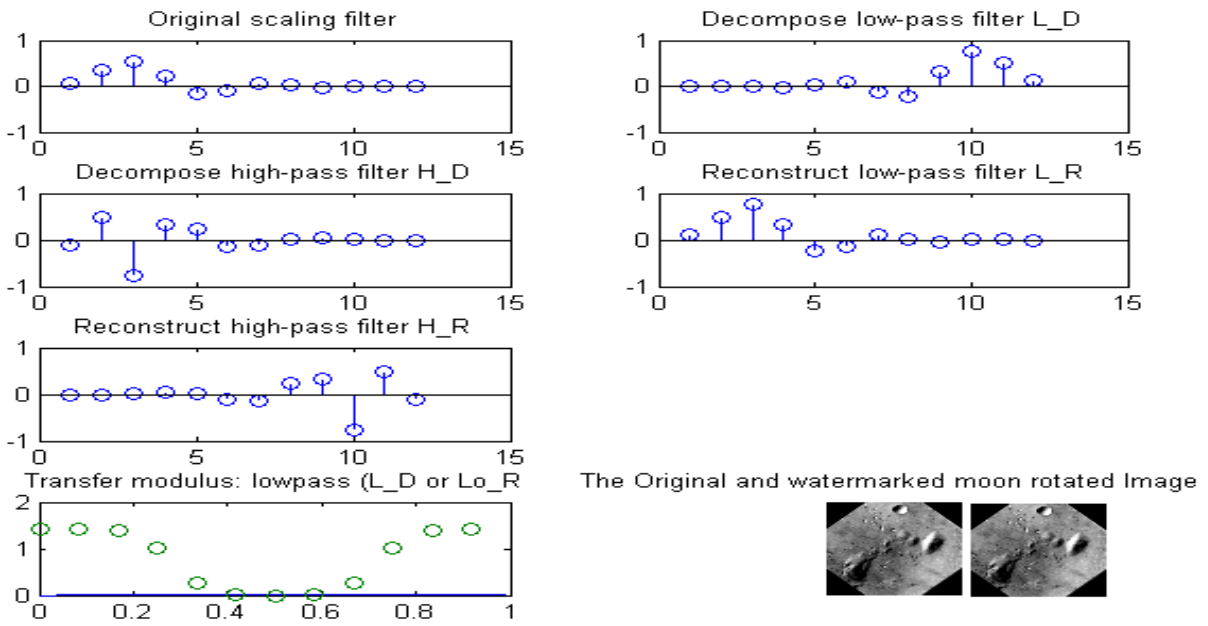

Figure 28. A similarity between coefficents of original watermarked image under rotated attacks: (a) Moon rotated attacks (12.4233 db), (273.5305 db). 

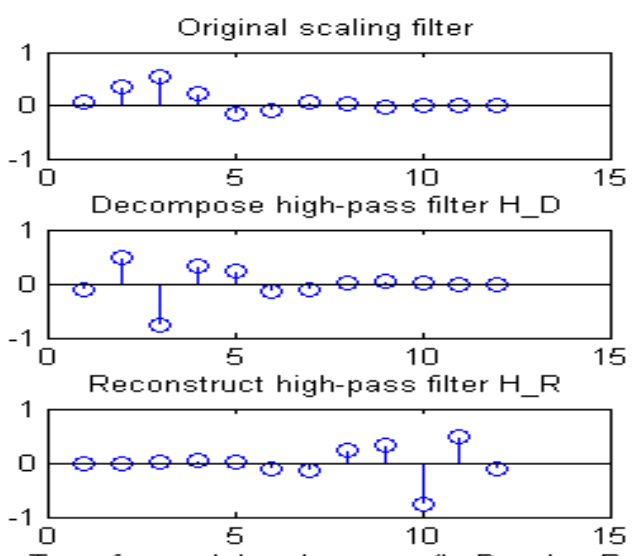

Transfer modulus: lowpass (L_D or Lo_R

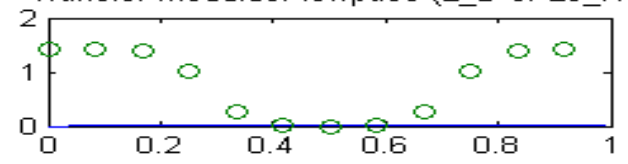

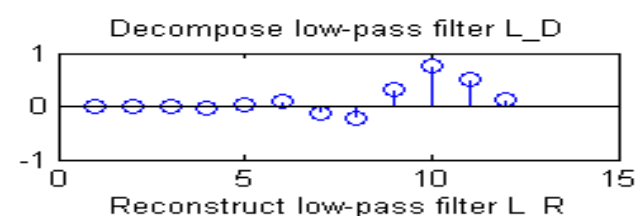

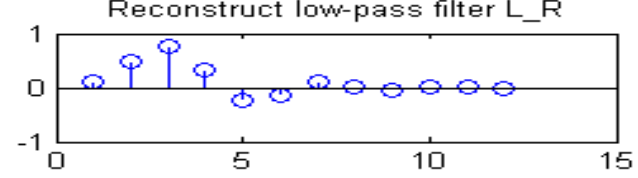

The Original and watermarked moon noise Image

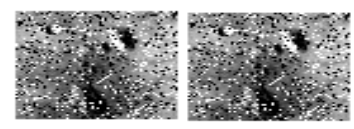

Figure 29. A similarity between coefficents of original watermarked image under noise attacks: (a) Moon noise attacks (11.1151 db), (274.1345 db).
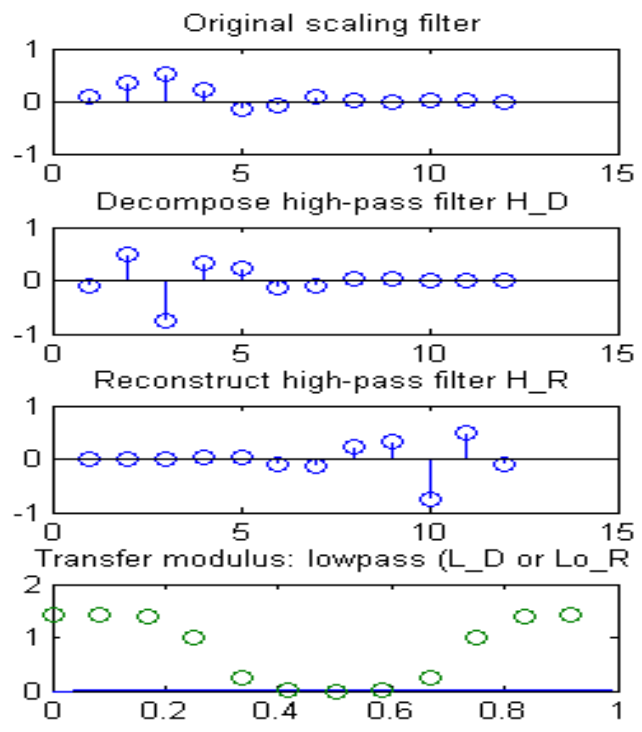

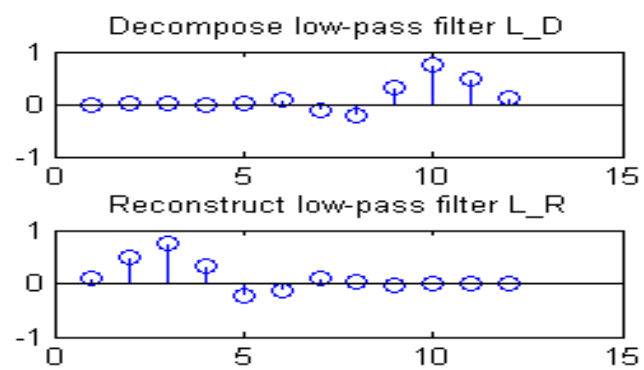

The Original and watermarked moon blurred Image

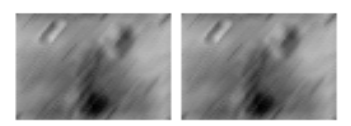

Figure 30. A similarity between coefficents of original watermarked image under without and blurred attacks: (a) Moon blurred attacks (23.5507 db), (276.7801 db).

In this way, we designed a singular value decomposition algorithm based on digital image watermarking technique and are to be following by this Figure 31.

\section{Conclusions}

Digital watermarking is one of emerging area of research. In this paper, we proposed a digital image watermarking algorithm based on singular value decomposition. The algorithm is used for watermarking embedding and watermark extraction. The feautre of the D component and the realation ship between the U Component coefficents were explored in the proposed technique that provide stronger robustness against different attacks and better image quality. So, Digital image watermarking techniques are secure on this algorithm. If alpha has a less than 0.2 value then quality of the original image and watermarked image is good. The experimental results also recognized the effectiveness of the proposed technique. Because of these properties, SVD is used for DCT, DFT, and DWT transformations, and one-way non-symmetrical decomposition. These provide the advantages of various sizes of transformation and more security. That is a good performance of the proposed scheme both in 


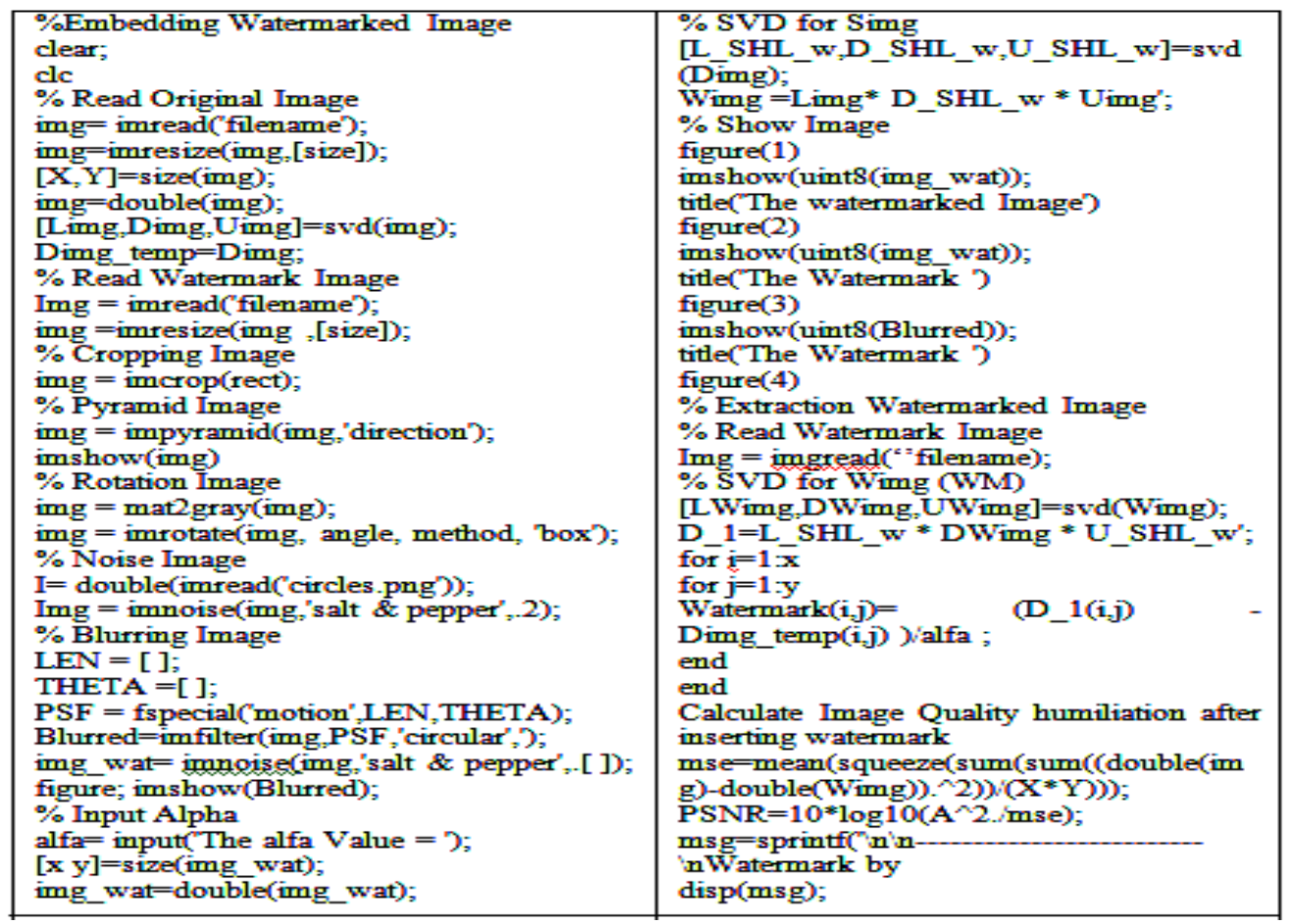

Figure 31. SVD algorithm

terms of robustness and security.

\section{References}

[1] D. Kundur and D. Hatzinakos, "Digital Watermarking Using Multiresolution Wavelet Decomposition," Speech and Signal Processing Proceedings, Acoustics, 1997, pp. 2969-2972.

[2] P. Zeng and C. Jin, "Image Adaptive Watermarking Using Visual Models," IEEE Journal on Selected Areas in Communications, Vol. 16, No. 4, 1998, pp. 525-539. doi:10.1109/49.668975

[3] L. Rajab, T. Khatib and A. Haj, "Combined DWT-DCT Digital Image Watermarking," Journal of Computer Science, Vol. 3, 2002, pp. 740-749.

[4] C. C. Chang and P. Tsai, "SVD-based Digital Image Watermarking Scheme," Pattern Recognition Letters, Vol. 26, No. 10, 2005, pp. 1577-1586. doi:10.1016/j.patrec.2005.01.004

[5] T. V. Nguyen and J. C. Patra, "A Simple ICA Based Digital Image Watermarking Scheme," Digital Signal Processing, Vol. 18, No. 5, 2007, pp. 762-776. doi:10.1016/j.dsp.2007.10.004

[6] A. H. Ali and M. Ahmad, "Digital Audio Watermarking Based on the Discrete Wavelets Transform and Singular Value Decomposition," Europe Journal of Science Research, Vol. 39, No. 1, 2010, pp. 6-21.

[7] C. I. Podilchuk and E. J. Delp, "Digital Watermarking: Algorithms and Applications," IEEE Signal Processing
Magazine, Vol. 18, No. 4, 2001, pp. 33-46. doi:10.1109/79.939835

[8] B. Kim, J. G. Choi and D. Min, "Robust Digital Watermarking Method Against Geometric Attacks," Real Time Imaging Processing, Vol. 9, No. 2, 2003, pp. 139-149. doi:10.1016/S1077-2014(03)00020-2

[9] H. Tina, W. Lu, R. prawn and Y. Ming, "A Fragile Watermarking Scheme for 3D Meshes," MM-SEC'05, ACM, pp. 117-123, 2008.

[10] W. Loo and X. Kingsbury, "Digital Watermarking using Complex Wavelets," International Conference on Image Processing, Vol. 3, 1999, pp. 29-32.

[11] M. Jiansheng and L. Sukang, "A Digital Watermarking Algorithm Based on DCT and DWT," International Symposium on Web Information System and Application (WISA), 2009, pp. 104-107.

[12] A. H. Ali and M. Ahmad, "Digital Audio Watermarking Based on the Discrete Wavelets Transform and Singular Value Decomposition," Europe Journal of Science Research, Vol. 39, No. 1, 2010, pp. 6-21.

[13] W. Lu, H. Lu and F. L. Chung, "Feature Based Watermarking Using Watermark Template Match," Applied Mathematics and Computation, Vol. 177, No. 1, 2011, pp. 886-893.

[14] Y. Lu, K. Uehira and K. Yanaka, "Practical Evaluation of Illumination Watermarking Technique Using Orthogonal Transforms," Journal of Display Technology, Vol. 6, No. 9, 2010, pp. 351-358. doi:10.1109/JDT.2010.2049336 